\title{
UPPSALA RADIOCARBON MEASUREMENTS VIII
}

\author{
ALLAN STENBERG and INGRID U. OLSSON
}

Fysiska Institutionen, Uppsala Universitet, Uppsala, Sweden

The following list covers the samples measured since autumn 1964, when Uppsala VI was written, to determine the increase of the $\mathrm{C}^{14} / \mathrm{C}^{12}$ ratio due to explosion of nuclear devices.

The technique used is the same as that previously described by Olsson (1958). The collection of $\mathrm{CO}_{2}$ is still made by static absorption in $0.5 \mathrm{~N} \mathrm{NaOH}$ as described earlier (Uppsala VI). The reference sample is $95 \%$ of the activity of the NBS oxalic-acid standard in the year 1950 . Corrections for deviations from the normal $\mathrm{C}^{13} / \mathrm{C}^{12}$ ratio are applied. No correction for industrial effect is applied. All results are given according to the Editorial Statement in Radiocarbon:

$$
\Delta=\delta \mathrm{C}^{14}-\left(2 \delta \mathrm{C}^{13}+50\right)\left(1+\frac{\delta \mathrm{C}^{14}}{1000}\right)
$$

\section{ACKNOWLEDGMENTS}

Sincere thanks are due to Prof. Gustav Sandberg, Uppsala, to Prof. Arne E. Sandström, Uppsala, and to Telegrafstyret, Oslo for giving us help and allowing us to collect samples at Abisko, onboard M/S Stratus and on Kapp Linné, Spitsbergen. Special thanks are due to Mr. Lennart Johansson and Mr. Karl Andersson (Abisko); fil.kand. Bert Sporre and fil.stud. Mats Nordström (Stratus); Radiobestyrer Kåre Henriksen and Mr. Oddvar Ulvang (Spitsbergen), who have helped us with the collection of $\mathrm{CO}_{2}$. Special thanks are also due to Dr. R. Ryhage and his coworkers for making the $\mathrm{C}^{13} / \mathrm{C}^{12}$ determinations; to Prof. K. Siegbahn, who has made it possible to do this work at the institute; and to Statens Naturvetenskapliga Forskningsråd, which has given the laboratory the financial support necessary for this project. The authors are indebted to Mrs. Maud Söderman for taking part in the routine work the whole time, Miss Birgitta Wallin and Miss Ditte Ekwall for assisting during part of the time. The authors also want to express their thanks to fil.kand. Enar Granström, who helped us for two months.

\section{A. Abisko, Sweden}

Abisko Naturvetenskapliga Station is scientific research station belonging to Kungliga Vetenskapsakademien. Sampling apparatus $\left(68^{\circ}\right.$ $20.5^{\prime} \mathrm{N}$ Lat, $18^{\circ} 49.3^{\prime} \mathrm{E}$ Long) is $390 \mathrm{~m}$ above sealevel near the lake Torne Träsk in mountain district of Sweden. Railway passing near is used by electrical trains (except one engine used at railway station). The few houses and the tourist station 0.2 to $1.5 \mathrm{~km}$ away are heated either with oil or wood. Due to absence of a road connection only a few motor vehicles are used. Thus contamination of the locality by fossil fuels is minimal. Apparatus is placed above level of tops of trees. 
Dating

\begin{tabular}{|c|c|c|c|c|c|c|c|}
\hline No. & No., & Year, & Month, & Day & $\delta \mathrm{C}^{14} / / c 0$ & $\delta \mathrm{C}^{13} / \omega_{0}$ & $\Delta \% c$ \\
\hline U-351. & UA-134s, & 1964 & July, & 5 to 8 & 986 & -24.1 & $867 \pm 13$ \\
\hline U-352. & UA-135, & 1964, & Sept, & 5 to 8 & 968 & -28.5 & $982 \pm 14$ \\
\hline U-368. & UA-138, & 1964 & Oct, & 15 to 18. & 894 & -24.4 & $892 \pm 23$ \\
\hline U-369. & UA-140, & 1964 & Nov, & 25 to 28. & 702 & -23.5 & $697 \pm 40$ \\
\hline $\mathrm{U}-370$. & UA-142, & 1964 & Dec, & 15 to 18. & 782 & -22.1 & $772 \pm 13$ \\
\hline U-371. & UA-143, & 1965 & Jan, & 15 to 18. & 756 & -25.2 & $756 \pm 13$ \\
\hline U-372. & UA-146, & 1965, & Febr, & 15 to 18. & 754 & -2 & $755 \pm 13$ \\
\hline U-373. & UA-149, & 1965 & Mar, & 15 to 18 & 737 & -2 & $743 \pm 17$ \\
\hline $\mathrm{U}-374$. & UA-152, & 1965 & April, & 15 to 18. & 748 & -28.2 & $759 \pm 13$ \\
\hline U-375. & UA-137s, & 1964 & Oct, & 5 to 8 & 918 & -27.0 & $925 \pm 14$ \\
\hline U-376. & UA-157, & 1965 & June, & 15 to 18. & 115 & -26.2 & $1155 \pm 15$ \\
\hline $\mathrm{U}-377$. & UA-160, & 1965 & July, & 15 to 18. & 1274 & -3 & $8 \pm 15$ \\
\hline U-378. & UA-139s, & 1964 & $\mathrm{Oct}$ & 25 to 28. & 892 & -2 & $0 \pm 14$ \\
\hline U-379. & UA-163, & 1965, & Aug, & 15 to 18. & 1034 & -24 & $1030 \pm 14$ \\
\hline U-386. & UA-165, & 1965 & Sept, & 15 to 18 & 1001 & -2 & $1003 \pm 17$ \\
\hline U-387. & UA-168, & 1965 & Oct, & $15 \mathrm{tc}$ & 92 & -2 & $928 \pm 16$ \\
\hline U-388. & UA-155, & 1965 & May, & 15 to 18. & 2066 & -2 & \pm 17 \\
\hline U-389. & UA- & 1966, & Jan, & 15 to 18. & 702 & -25.4 & \pm 15 \\
\hline U-390. & UA-173, & 1965 & Dec, & $15 \mathrm{tc}$ & 773 & -2 & $775 \pm 13$ \\
\hline-391 & UA-171, & 1965 & Nov, & $15 \mathrm{tc}$ & 786 & -2 & $794 \pm 13$ \\
\hline-392. & UA-187, & 1966 & May, & 5 to 8 & 687 & -27.2 & $694 \pm 21$ \\
\hline U-393. & UA-153s, & 1965, & April, & 25 to 28. & 35160 & -25.8 & $35220 \pm 140$ \\
\hline U-394. & UA-161s, & 1965 & July, & 25 to 28. & 1038 & $-25.8^{x}$ & $(1041 \pm 19)$ \\
\hline-2300. & UA-154s, & 1965, & May, & 5 to 8 & & -24.1 & $6954 \pm 52$ \\
\hline-2301. & UA-161s, & 1965, & July, & 25 to 28. & 1068 & $-25.8^{x}$ & $(1072 \pm 40)$ \\
\hline-2304. & UA-179, & 1966 , & Feb, & 15 to 18. & 641 & -25.3 & $642 \pm 12$ \\
\hline U-2305. & UA-181, & 1966, & Mar, & 5 to 8 & 643 & -25.5 & $645 \pm 11$ \\
\hline-2306. & UA-182, & 1966 & Mar, & 15 to 18. & 721 & -26.4 & $722 \pm 15$ \\
\hline-2307. & UA-159s, & 1965 & July, & 5 to 8 & 1420 & -21.4 & $1403 \pm 23$ \\
\hline 2308 . & UA-156s, & 1965, & June, & 5 to 8 & 1259 & -25.4 & $1261 \pm 14$ \\
\hline 2309. & UA-189, & 1966 & May, & 15 to 18. & 700 & -27.7 & $709 \pm 15$ \\
\hline-2310. & UA-191, & 1966, & June, & 15 to 18. & 721 & -25.3 & $722 \pm 13$ \\
\hline-2311. & UA-184, & 1966, & April, & 5 to 8. & 744 & -25.6 & $746 \pm 15$ \\
\hline-2312. & UA-190, & 1966 & May, & 25 to 28 . & 674 & -26.5 & $679 \pm 12$ \\
\hline
\end{tabular}

$\mathrm{x}_{\delta \mathrm{C}^{13}}$ assumed

Erratum in Uppsala VI p. 332, read:

$\begin{array}{llllll}\text { U-1305. UA-118, 1964, Jan, } 15 \text { to } 18 & 761 & -25.5 & 763 \pm 10\end{array}$

\section{B. Kapp Linné, Spitsbergen}

Kapp Linné is a radiostation and meteorologic station belonging to Telegrafstyret, Oslo, Norway. Sampling apparatus $\left(78^{\circ} 04^{\prime} \mathrm{N}\right.$ Lat, 
$13^{\circ} 38^{\prime}$ E Long) is only a few meters above sealevel near shore at mouth of Isfjorden. Apparatus is placed on top of a small house rather far from the generators and their smoke.

\begin{tabular}{ccclllll}
$\begin{array}{c}\text { Dating } \\
\text { No. }\end{array}$ & No., & Year, & Month, & Day & $\delta \mathrm{C}^{14 \%} \%$ & $\delta \mathrm{C}^{13} / \mathrm{cc}$ & $\Delta^{\prime}$ c \\
\hline U-360. & US-31, & 1964, & Jan, & 15 to 18. & 799 & -30.8 & $820 \pm 15$ \\
U-380. & US-35, & 1964, & July, & 27 to 31. & 967 & -24.7 & $966 \pm 14$ \\
U-381. & US-36, & 1964, & Aug, & 23 to 27. & 957 & -27.8 & $968 \pm 14$ \\
U-382. & US-37, & 1964, & Sept, & 15 to 19. & 925 & -22.8 & $917 \pm 14$ \\
U-383. & US-38, & 1964, & Oct, & 20 to 24. & 895 & -26.2 & $900 \pm 13$ \\
U-384. & US-40, & 1965, & June, & 23 to 27. & 809 & -26.5 & $814 \pm 13$ \\
U-385. & US-41, & 1965, & Aug, & 23 to 27. & 814 & -25.8 & $(820 \pm 13)$ \\
U-2313. & US-45, & 1965, & Dec, & 19 to 23. & 732 & -24.9 & $732 \pm 12$ \\
U-2314. & US-46, & 1966, & Jan, & 22 to 26. & 726 & -28.7 & $739 \pm 14$ \\
U-2316. & US-44, & 1965, & Nov, & 22 to 26. & 799 & -23.5 & $793 \pm 17$
\end{tabular}

$\mathrm{x}_{\delta} \mathrm{C}^{13}$ assumed

\section{C. $M / S$ Stratus}

During 1964, $\mathrm{CO}_{2}$ was collected onboard $\mathrm{M} / \mathrm{S}$ Stratus by scientists taking care of the apparatus for registering the cosmic rays. $\mathrm{CO}$. was collected in a bucket. This was put at various places on the ship to minimize contamination from the chimney. Collection periods were usually limited to one or two days, but this proved to be too short a time to get enough $\mathrm{CO}_{2}$ for normal filling of proportional counter. As a consequence of this most samples had to be diluted.

General Comment: from Figure 1 it is obvious that increase in activity usually is $1 / 2$ to 1 month later in Spitsbergen than Abisko during the last summers. The high peak in Abisko starting in the end of April 1965 seems to be a local event. It will be discussed at I.A.E.A. symposium in Monaco, 1967. The 1964 values from Southern hemisphere are lower than those from Northern hemisphere. Our values have partly been discussed previously (Olsson et al., 1966). Reference list contains some recent papers not included in Uppsala VI.

Date List:

\section{REFERENCFS}

Uppsala VI Olsson and Karlén, 1965

Berger, Rainer, Fergusson, G. J., and Libby, W. F., 1965, UCLA radiocarbon dates IV: Radiocarbon, v. 7, p. 336-371.

Fergusson, G. J., 1965, Radiocarbon and tritium in the upper troposphere: Internat. Conf. Radiocarbon and Tritium Dating, 6th, Pullman, Washington, June 7-11, 1965, Proc., p. 525-540.

Lal, D., and Rama, 1966, Characteristics of global tropospheric mixing based on manmade $\mathrm{C}^{14}, \mathrm{H}^{3}$, and $\mathrm{Sr}^{80}$ : Jour. Geophys. Research, v. 71, p. 2865-2874.

Machta, L., 1958, Global scale dispension by the atmosphere: U.N. Internat. Conf. on the Peaceful Uses of Atomic Energy, 2nd, Geneva, Proc., no. 18, p. 519-525. 
Münnich, K. O., and Vogel, J. C., 1963, Investigation of meridional transport in the troposphere by means of carbon-14 measurements: Radioactive Dating, I.A.E.A., Vienna 1963, p. 189-197.

Nydal, Reidar, and Lövseth, Knut, 1965, Distribution of radiocarbon from nuclear tests: Nature, v. 206, no. 4988, p. 1029-1031.

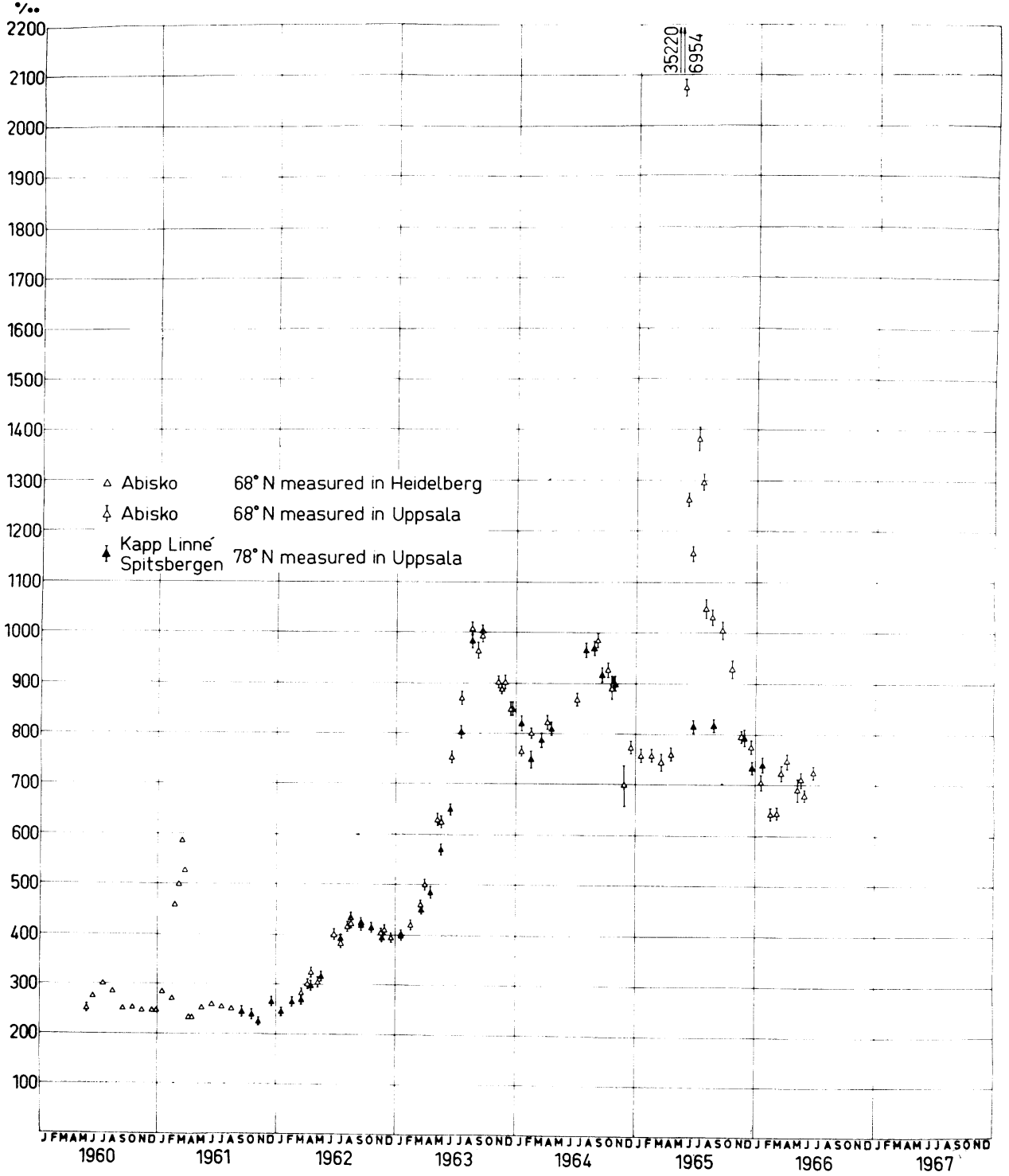

Fig. 1. The per mil $\mathrm{C}^{14}$ excess over natural concentration $(\Delta)$ at Abisko and Kapp Linné. Points given with statistical errors are determined at the Uppsala $\mathrm{C}^{14}$ lab. Points given without statistical errors are determined at the Heidelberg $\mathrm{C}^{14} \mathrm{lab}$ (Münnich and Vogel, 1963) but collected through the Uppsala lab. 


\begin{tabular}{|c|c|c|c|c|c|c|c|c|c|c|c|}
\hline & 20 & के & $\stackrel{2}{\sim}$ & कo & 2 & 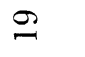 & $\stackrel{\sim}{ }$ & $\stackrel{2}{=}$ & 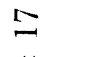 & $\stackrel{V}{-}$ & $\stackrel{2}{=}$ \\
\hline 2 & $H$ & +1 & +1 & +1 & +1 & +1 & +1 & +1 & +1 & +1 & +1 \\
\hline$\triangleleft$ & $\begin{array}{l}\infty \\
\infty \\
\infty\end{array}$ & $\frac{9}{10}$ & $\vec{F}$ & $\begin{array}{l}\infty \\
\stackrel{2}{1}\end{array}$ & $\stackrel{\infty}{\stackrel{\infty}{\Omega}}$ & 䒿 & $\stackrel{\infty}{b}$ & $\stackrel{\hat{R}}{\hat{R}}$ & $\underset{10}{8}$ & $\overline{\widehat{O}}$ & $\stackrel{8}{0}$ \\
\hline نَ & $\stackrel{r}{\stackrel{r}{N}}$ & $\begin{array}{l}\stackrel{9}{0} \\
\stackrel{0}{1} \\
1\end{array}$ & 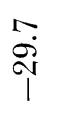 & $\underset{\stackrel{H}{N}}{\stackrel{O}{r}}$ & $\begin{array}{c}o \\
\stackrel{9}{1} \\
\stackrel{1}{1}\end{array}$ & 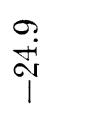 & $\begin{array}{l}\infty \\
\stackrel{\infty}{a} \\
\stackrel{1}{1}\end{array}$ & $\begin{array}{l}\infty \\
2 \\
\stackrel{2}{\sigma} \\
1\end{array}$ & $\underset{\dot{T}}{\stackrel{0}{r}}$ & $\begin{array}{l}\infty \\
\underset{N}{N} \\
i\end{array}$ & $\begin{array}{l}20 \\
20 \\
19 \\
1\end{array}$ \\
\hline שِ & $\begin{array}{l}\infty \\
\text { a } \\
\infty\end{array}$ & 帘 & 点 & $\underset{N}{F}$ & $\begin{array}{l}\mathscr{D} \\
\qquad \infty\end{array}$ & $\begin{array}{l}10 \\
10 \\
10\end{array}$ & J & $\stackrel{g}{g}$ & O̊̀ & 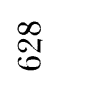 & $\begin{array}{l}\infty \\
\varnothing\end{array}$ \\
\hline$\Xi$ & 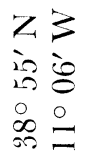 & 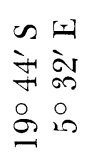 & 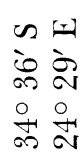 & 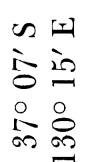 & 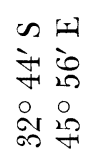 & 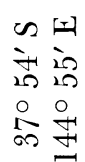 & 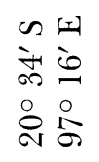 & $\begin{array}{l}z 3 \\
\text { is is } \\
\text { o i } \\
\text { os } \\
\text { @ }\end{array}$ & 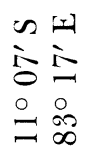 & 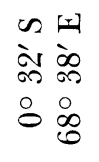 & $\begin{array}{ll}7 & 1 \\
0 & 1 \\
0 & 8 \\
0 & 0 \\
0 & 0 \\
\text { in }\end{array}$ \\
\hline $\overrightarrow{0}$ & $\begin{array}{cc}1 & 1 \\
\text { if is } \\
\text { is } \\
\text { is } \\
0 \\
0\end{array}$ & $\begin{array}{ll}1 & 1 \\
\text { is } & 1 \\
\text { a } & \text { o } \\
0 & 0 \\
\text { on } & 0\end{array}$ & 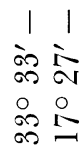 & $\begin{array}{cc}1 & 1 \\
\infty & 1 \\
0 & 0 \\
0 & 0 \\
\infty & \infty \\
\infty & \infty \\
\infty & \infty\end{array}$ & \begin{tabular}{cc}
1 & 1 \\
के & \multicolumn{2}{c}{} \\
0 & $\infty$ \\
0 & 0 \\
0 & 0
\end{tabular} & $\begin{array}{cc}1 & 1 \\
j & \infty \\
\forall & 0 \\
0 & 0 \\
10 & \infty \\
0 & 0\end{array}$ & $\begin{array}{ll}1 & 1 \\
\text { oे } & \text { oे } \\
0 & 0 \\
\text { o } & 8 \\
\text { o } & 8\end{array}$ & 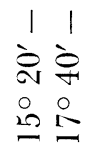 & 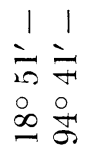 & $\begin{array}{cc}1 & 1 \\
\text { in } & \text { i } \\
\text { in } & \text { in } \\
0 & 0 \\
\infty & 0\end{array}$ & \begin{tabular}{ll}
1 & 1 \\
is & \multicolumn{1}{c}{} \\
is \\
0 \\
0
\end{tabular} \\
\hline $\overrightarrow{\tilde{\theta}}$ & 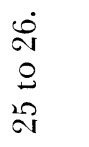 & $\begin{array}{c}\dot{0} \\
0 \\
0 \\
20\end{array}$ & $\begin{array}{l}\dot{0} \\
\stackrel{0}{\sigma} \\
\dot{\sigma}\end{array}$ & $\begin{array}{r}\dot{+} \\
\stackrel{0}{0} \\
\infty\end{array}$ & $\begin{array}{l}\frac{0}{0} \\
\stackrel{0}{0}\end{array}$ & $\begin{array}{c}\dot{v} \\
\stackrel{\infty}{a} \\
\stackrel{\infty}{n}\end{array}$ & $\begin{array}{l}\dot{0} \\
0 \\
0 \\
0\end{array}$ & $\begin{array}{l}\dot{+} \\
\stackrel{9}{v} \\
\stackrel{v}{*}\end{array}$ & $\begin{array}{l}\dot{\varrho} \\
\stackrel{\Xi}{\Xi}\end{array}$ & $\begin{array}{l}\stackrel{\infty}{2} \\
\stackrel{0}{=}\end{array}$ & $\begin{array}{l}20 \\
20 \\
0 \\
0\end{array}$ \\
\hline & $\hat{\tilde{\Xi}}$ & $\dot{0}$ & i & $\stackrel{\overrightarrow{\mathrm{J}}}{\mathrm{z}}$ & $\stackrel{\infty 00}{\sum_{4}}$ & 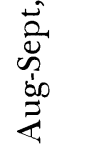 & zे & $\stackrel{\dot{00}}{\Xi}$ & $\vec{z}$ & $\vec{z}$ & हे \\
\hline$\stackrel{\tilde{\Xi}}{\tilde{\nu}}$ & 焉 & $\stackrel{d}{\mathscr{D}}$ & 递 & $\stackrel{\mathbb{S}}{\Phi}$ & 苞 & 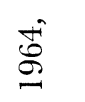 & $\stackrel{d}{\mathscr{S}}$ & 递 & $\vec{E}$ & $\stackrel{\vec{\Xi}}{\mathscr{E}}$ & 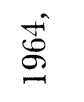 \\
\hline$\dot{\dot{z}}$ & $\hat{-i}$ & $\infty$ & 20 & $\therefore$ & $\infty$ & $\sigma^{\circ}$ & $\stackrel{0}{0}$ & $\hat{=}$ & 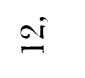 & 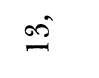 & $\ddot{ \pm}$ \\
\hline & $\begin{array}{l}\infty \\
\infty \\
\infty \\
0 \\
\vdots \\
\vdots\end{array}$ & $\begin{array}{l}10 \\
10 \\
00 \\
\dot{0} \\
\dot{0}\end{array}$ & $\begin{array}{l}10 \\
100 \\
\text { 10 } \\
\dot{0}\end{array}$ & 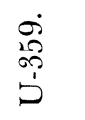 & $\begin{array}{l}\dot{D} \\
\dot{D} \\
\dot{D}\end{array}$ & $\begin{array}{l}\text { ôं } \\
\stackrel{b}{0} \\
\dot{\nu}\end{array}$ & 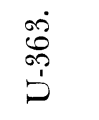 & $\begin{array}{l}\dot{D} \\
\infty \\
\dot{D}\end{array}$ & $\begin{array}{l}10 \\
0 \\
0 \\
\dot{0} \\
\dot{D}\end{array}$ & $\begin{array}{l}\dot{0} \\
\dot{0} \\
\dot{D}\end{array}$ & $\begin{array}{l}\dot{0} \\
\infty \\
\dot{0}\end{array}$ \\
\hline
\end{tabular}


Nydal, Reidar, 1966, Variation in $\mathrm{C}^{14}$ concentration in the atmosphere during the last several years: Tellus, v. 18, no. 2-3, p. 271-279.

Olsson, I. U., 1958, A $\mathrm{C}^{14}$ dating station using the $\mathrm{CO}_{2}$ proportional counting method: Arkiv för Fysik, v. 13, p. 37-60.

Olsson, I. U., and Karlén, Ingvar, 1965, Uppsala radiocarbon measurements VI: Radio(arbon, v. 7, p. 331-335.

Olsson, I. U., Karlén, Ingvar, and Stenberg, Allan, 1966, Radiocarbon variations in the atmosphere: Tellus, v. 18, p. 293-297.

Rafter, T. A., 1965, Carbon-14 variations in Nature. Part 2 -Increase in ${ }^{14} \mathrm{C}$ activity in the atmosphere of the southern hemisphere from the testing of nuclear weapons: New Zealand Jour. of Sci., v. 8, no. 4, p. 472-493.

Lnited Nations Scientific Committee on the Effects of Atomic Radiation, 1964, General Assembly official records, 19th session, Supp. No. 14 (A/5814). 